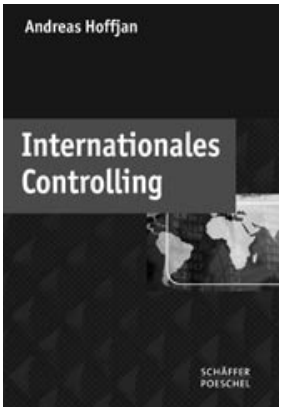

Prof. Dr.

Andreas Hoffjan

\section{Internationales Controlling}

\section{Schäffer-Poeschel Verlag}

Stuttgart 2009

311 Seiten, 39,95€

ISBN: 978-3-7910-2532-2

Ausgehend von der zunehmend globalisierten wirtschaftlichen Entwicklung behandelt das vorliegende Buch das Controlling in internationalen Unternehmen. Besondere Widmung erfahren diejenigen Prozesse, die durch die internationale Dimension multinationaler Unternehmen eine besondere Herausforderung für das Controlling darstellen. Die Zielsetzung des Buches liegt darin, die Grundlagen des Controllings international tätiger Unternehmen darzustellen, wie auch Einzelprobleme der Entscheidungsunterstützung im Auslandsgeschäft zu analysieren.

Im ersten Teil des Buches beschreibt Hoffjan zunächst den „deutschen“ Controlling-Begriff und grenzt diesen anschließend von international vergleichbaren Begrifflichkeiten ab. Anschließend wird durch die Gegenüberstellung der Controlling-Praktiken im deutsch- wie englischsprachigen Raum die Grundlage für die nachfolgenden Kapitel des Buches gelegt. Zunächst wird der Begriff „Management Accounting “ als Pendant des deutschsprachigen „Controlling“ eingeführt. Gestützt auf die Erkenntnisse einer Vielzahl deutscher und internationaler empirischer Studien geht Hoffjan auf die Unterschiede zwischen Controllern und Management Accountants ein, vergleicht deren Aufgaben, Adressaten, und die jeweilige hierarchische Stellung innerhalb der Unternehmen. Ein zentraler Unterschied zwischen beiden Gruppen besteht darin, dass in Deutschland aufgrund des Zweikreissystems die Daten des externen Rechnungswesen von denen des Controlling getrennt werden, während im angloamerikani- schen Raum meist eine einheitliche Datenbasis für externe und interne Zwecke genutzt wird.

Der zweite Teil des Buches widmet sich Störfaktoren im internationalen Controlling. Hoffjan stellt vor, wie sich länderspezifische Unterschiede, beispielweise die Besonderheiten im Bereich der Landeskultur, Währungsunterschiede oder verschiedene Steuersysteme auf das Controlling auswirken. Trotz der in den letzten Jahren festgestellten zunehmenden Harmonisierung von Controlling-Prozessen in internationalen Unternehmen, werden kulturelle Differenzen zwischen Ländern weiterhin bestehen bleiben. Die von Hoffjan präsentierten Konzepte zur Untersuchung kultureller Unterschiede, z. B. nach Hofstede, erweisen sich dabei als nützlich, kulturelle Distanzen zu erkennen, zu analysieren, und zu minimieren. Abschließend wird die internationale Steuerplanung, der in internationalen Unternehmen eine exponierte Stellung zukommt, vorgestellt. Verrechnungspreise, die innerkonzernliche Leistungstransfers abbilden sollen, sorgen in diesem Zusammenhang für erhöhte Komplexität. Einerseits sollen sie dazu beitragen, die Konzernsteuerquote zu minimieren, andererseits müssen die verwendeten Größen auch von nationalen Finanzverwaltungen akzeptiert werden. Vor diesem Hintergrund wird die enge $\mathrm{Zu}$ sammenarbeit zwischen (zentralen) Controllingabteilungen und Steuerabteilungen als unumgänglich herausgestellt. Dafür spricht auch, dass es aufgrund der Mannigfaltigkeit von Steuersystemen und Konzernstrukturen keine „Best Practice“Empfehlung hierfür gibt.

Im dritten Teil des Buches, „Erfolgsmessung “, beschäftigt sich der Autor mit den Besonderheiten der internationalen Geschäftstätigkeit im Rahmen der wertorientierten Steuerung ausländischer Tochtergesellschaften. Herausforderungen ergeben sich hier aufgrund politischer Risiken oder makroökonomischer Faktoren wie z.B. Inflation. Eine umfassende Kenntnis nationaler Rechnungslegungsvorschriften und -methoden ist - laut Hoffjan - zwingend erforderlich, um ggf. verzerrte Informationen korrekt anzupassen und $\mathrm{zu}$ interpretieren. Als besonders geeignetes Instrument für die Erfolgsmessung in internationalen Unternehmen wird die Balanced Scorecard thematisiert, welche wesentliche Unzulänglichkeiten „klassischer" Methoden durch die Einbeziehung nicht-finanzieller Kennzahlen überwindet. Als „Wehrmutstropfen“ für die Implementierung der Balanced Scorecard wird auf eventuelle Akzeptanzprobleme des Konzeptes aufgrund großer kultureller Unterschiede hingewiesen.

Der vierte Teil des Buches ist der Relevanz des internationalen Controllings bei strategischen Entscheidungen gewidmet. Dazu wird anhand von Praxis-Beispielen aus den Bereichen „Offshoring“ und Personalcontrolling erörtert, welche Rolle das internationale Controlling in der Entscheidungsfindung einnimmt. Der Autor hält es z. B. für ratsam, das Controlling nicht zu spät in Offshoring-Entscheidungen einzubeziehen, wenn u.U. nur noch strategische Handlungsalternativen, z. B. die Rückverlagerung, zur Verfügung stehen. Im Zusammenhang mit der Entsendung wird darauf aufmerksam gemacht, dass kulturelle Unterschiede und ihre Auswirkungen auf Personen, nur schwerlich ,einzuplanen“ sind.

Zum Schluss beschäftigt sich Hoffjan mit der Organisation des internationalen Controllings. Speziell geht er auf das Spannungsfeld zwischen dem Bedürfnis nach weltweiter Standardisierung und der Notwendigkeit nationaler Anpassung ein. Er stellt unterschiedliche Konzepte und Kontextfaktoren vor, deren Analyse internationalen Unternehmen dabei helfen kann, sich in diesem Spannungsfeld möglichst reibungslos zu bewegen. Der Autor schließt mit der Feststellung, dass der zunehmende Wettbewerbsdruck und die Notwendigkeit global mit hoher Schnelligkeit zu handeln, zwar eine Standardisierung nötig machen, jedoch auch der nötigen Differenzierung „Vor Ort“ in ausreichendem Maße Rechnung getragen werden muss.

Fazit: Das vorliegende Buch eignet sich gleichermaßen für Studenten, die einen umfassenden Einblick in das internationale Controlling gewinnen möchten, wie auch für interessierte Praktiker, denen viele Beispiele aus der Unternehmenspraxis und Handlungsempfehlungen mitgeliefert werden. Vereinzelt lohnt sicherlich ein Blick in eine der angegebenen Quellen, da aufgrund des großen Umfangs der behandelten Themen eine hohe Detailtiefe naturgemäß nicht überall vorhanden ist. In diesem Zusammenhang sei auch auf vertiefende Lehrmaterialien zum Buch unter www.sp-dozenten.de hingewiesen (Registrierung erforderlich). Kurzum: Ein lesenswertes Buch und eine uneingeschränkte Weiterempfehlung!

Jochen Rehring, Vallendar 